Reprod. Nutr. Dévelop., 1985, 25 (1 B), 189-196.

\title{
Rôle des précurseurs d'adipocytes dans l'installation d'une obésité nutritionnelle avant le sevrage
}

\author{
Isabelle DUGAIL, Francine DUPUY, Annie QUIGNARD-BOULANGÉ
}

Physiopathologie de la Nutrition, INSERM U.177, Institut biomédical des Cordeliers,

15, rue de l'Ecole de Médecine, 75270 Paris Cedex 06.

Summary. Role of adipocyte precursors in the initiation of nutritional obesity before weaning.

The stroma vascular fraction of adipose tissue partly consists of adipose precursor cells which can convert into adipocytes in vitro. The aim of this study was to investigate the possible contribution of cells from the stroma vascular compartment to the initiation of obesity induced by overfeeding during the early neonatal weeks in rats. Overfeeding during the suckling period was obtained by reducing the litter size.

The inguinal adipose tissue of overfed rats raised in litters of 4 pups each was overdeveloped compared to that of controls raised in litters of 8 pups each, and the difference between the two groups became significant as early as 10 days of age. At this age, adipose tissue enlargement was only due to adipocyte hypertrophy; afterwards, hyperplasia of the mature fat cells contributed to the overdevelopment of adipose tissue in 15-day old overfed rats. The cell number in the stroma vascular fraction increased in the overfed group as early as 10 days of age and thus preceded the onset of mature fat cell hyperplasia. The developmental pattern of lipoprotein lipase, glycerol-3-phosphate dehydrogenase, glycerol-acyl-transferase and acyl-CoA ligase activities in stromal cells did not depend on litter size, but specific enzyme activities were increased in 10-day old overfed rats compared to the controls.

These results indicate that early overfeeding induced cell proliferation in the stroma vascular compartment and also induced the enzyme activities involved in adipose conversion to increase in these cells. This strongly suggests that precursor cell differentiation was greater in overfed rats than in control rats.

\section{Introduction.}

II est possible de provoquer une suralimentation précoce chez le rat en réduisant la taille des portées dès la naissance (Oscai et Mc Garr, 1978). Lorsque les rats sont élevés par portées de taille réduite, leur croissance est plus rapide que lorsque les portées sont de taille standard (8-12 petits) (Kennedy, 1957 ; Widdow- 
son et Mac Cance, 1960 ; Cryer et Jones, 1979). De plus il a été montré que cette suralimentation précoce provoquait aussi une obésité durable (Knittle et Hirsch, 1968) ; en effet, les animaux élevés par petites portées développent à l'âge adulte une obésité persistante et caractérisée par une hypertrophie et une hyperplasie adipocytaire.

On sait que le tissu adipeux est composé de différents types cellulaires et après dissociation par la collagénase du tissu adipeux on peut séparer 2 compartiments cellulaires : les adipocytes matures et la fraction stroma-vasculaire qui est en partie constituée de cellules précurseurs, capables de se différencier en adipocytes avec une probabilité qui dépend à la fois de l'âge du donneur et de la localisation adipeuse (Djian et al., 1983). In vitro, des études portant soit sur des préadipocytes en culture primaire, soit sur des lignées préadipocytaires, ont montré que la différenciation se manifeste par l'apparition d'activités enzymatiques caractéristiques de la fonction adipocytaire comme la lipoprotéine lipase et la glycérol-3-phosphate-déshydrogénase (Hausman et al., 1980) qui sont de ce fait considérées comme des marqueurs précoces de différenciation.

Le rôle des précurseurs d'adipocytes dans l'installation d'une obésité induite par suralimentation précoce est à ce jour inconnu. Le but de ce travail est donc de savoir si la suralimentation précoce peut influencer la vitesse de réplication et/ou de différenciation des cellules précurseurs d'adipocytes de la fraction stromale. Pour ce faire, nous avons entrepris une étude longitudinale du compartiment stromal du tissu adipeux inguinal, qui se développe dans les premières heures suivant la naissance, et nous avons suivi, pendant la période de suralimentation, l'évolution des activités enzymatiques caractéristiques de la fonction adipocytaire dans ces cellules.

\section{Matériel et méthodes.}

Les animaux utilisés dans cette étude sont des rats élevés dans notre animalerie. Les femelles allaitantes sont gardées en cage individuelle et ont librement accès à la nourriture (biscuits de laboratoire UAR) et à l'eau de boisson. A l'âge de 2 jours, les ratons sont répartis au hasard pour former des portées de 4 ou 8 petits. A 2, 5, 10 et 15 jours, les animaux sont sacrifiés par décapitation et les pannicules de tissu adipeux inguinal des animaux provenant d'une même portée sont prélevés et poolés. Les tissus sont dissociés par la collagénase selon la méthode de Rothblatt et de Martinis (1977). Après dissociation et passage sur un filtre de nylon dont la taille des mailles est de $190 \mu \mathrm{m}$, les cellules adipeuses remontent à la surface après quelques minutes. La phase liquide inférieure est alors éliminée par aspiration et les adipocytes sont lavés 2 fois avec la solution saline de Hanks. La phase inférieure et les rinçages sont poolés et centrifugés 5 min à $360 \mathrm{~g}$. Le culot obtenu (cellules de la fraction stroma-vasculaire) est resuspendu dans un milieu approprié aux dosages enzymatiques.

Le volume des adipocytes a été déterminé par une méthode automatique en utilisant un Coulter counter (ZBI-C1000 channelyser) équipé d'un tube à orifice de $200 \mu \mathrm{m}$ de diamètre. Le nombre des adipocytes du tissu est calculé par le rapport 
du poids de lipides du tissu au poids moyen d'un adipocyte. Le contenu en lipides du tissu est mesuré sur fragments du pool tissulaire par gravimétrie après extraction des lipides par la méthode de Folch et al. (1957). Le nombre total de cellules stromales du tissu a été mesuré au Coulter counter équipé d'un tube à orifice de $100 \mu \mathrm{m}$ de diamètre. Les activités enzymatiques : lipoprotéine lipase (LPL), glycérol-3-phosphate deshydrogénase (GPDH), acyl-CoA ligase et glycérol-3-phosphateacyl-transférase (GPAT) ont été déterminées dans les cellules stromales. Pour chaque enzyme, exception faite de la LPL, les cellules sont broyées dans le tampon approprié et centrifugées $15 \mathrm{~min}$ à $1500 \mathrm{~g}$ : le surnageant est alors utilisé pour le dosage de la GPDH selon la technique de Kozak et Jensen (1974) modifiée par Wise et Green (1979), de la GPAT selon la méthode de Jamdar et Fallon (1973) et de l'acyl-CoA ligase selon la méthode de Tanaka et al. (1979). La lipoprotéine lipase est mesurée dans un homogénat après sonication des cellules dans un tampon $\mathrm{NH}_{4} \mathrm{Cl}-\mathrm{NH}_{4} \mathrm{OH} 50 \mathrm{mM}, \mathrm{pH} 8,1$ contenant $4 \mathrm{UI} / \mathrm{ml}$ d'héparine en utilisant un substrat radioactif (Nilsson-Ehle et Schotz, 1976). Les résultats sont exprimés en nmoles de produit formé par minute et par mg de protéines dans l'homogénat, les protéines étant dosées selon la méthode de Lowry et al. (1951).

\section{Résultats.}

La figure 1 montre l'évolution pendant les deux premières semaines de la vie du poids corporel et du poids du tissu adipeux inguinal chez les ratons élevés par petites portées ou par portées de taille standard (4 ou 8 petits). Dès l'âge de 10 jours, les animaux suralimentés sont significativement plus lourds que les témoins $(+16 \%)$ et cette différence s'accentue avec l'âge (+ $25 \%$ à 15 jours).
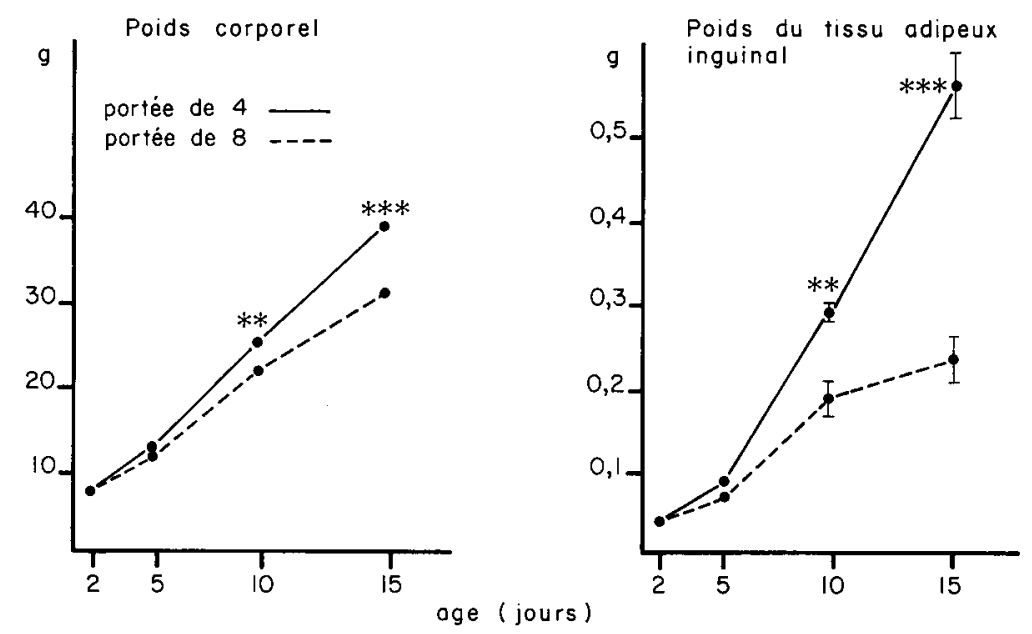

FIG. 1. - Effet de la suralimentation précoce sur le poids corporel et sur le poids du tissu adipeux inguinal. Chaque point représente une valeur moyenne \pm son écart type, calculée sur au moins 7 portées pour chaque groupe. Entre les 2 groupes, les valeurs moyennes sont comparées à l'aide du test $t$ de Student Fischer. Pour les valeurs statistiquement différentes, les niveaux de signification sont les suivants : ** $P<0,01 ;{ }^{* *} P<0,001$. 
Le développement du tissu adipeux inguinal est beaucoup plus rapide chez les animaux suralimentés que chez les témoins comme le montre l'évolution pondérale de ce tissu (fig. 1). Entre 5 et 15 jours, l'augmentation du poids du tissu adipeux est d'environ $47 \mathrm{mg} / \mathrm{j}$ pour 2 pannicules contre $16 \mathrm{mg} / \mathrm{j}$ chez les témoins. L'évolution de la taille et du nombre des adipocytes dans le tissu inguinal est représentée sur la figure 2. Pour les deux groupes d'animaux, la taille des adipocytes augmente entre 2 et 10 jours mais reste stable ensuite. Cependant, dès l'âge de 10 jours, la taille des adipocytes est significativement plus grande chez les animaux suralimentés que chez les témoins. Le nombre d'adipocytes dans le tissu inguinal augmente de façon identique dans les 2 groupes entre 5 et 10 jours
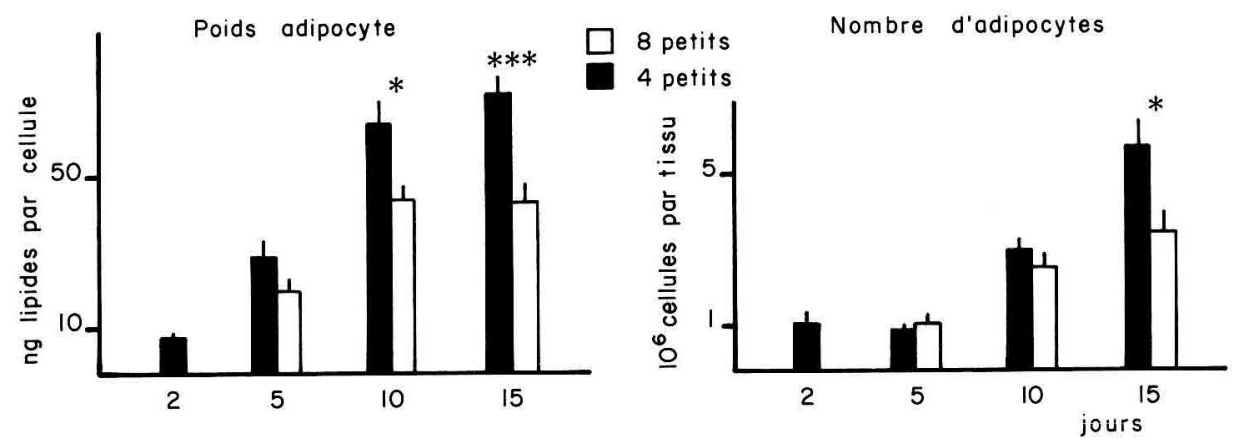

FIG. 2. - Influence de la taille de la portée sur la cellularité du tissu adipeux inguinal. Chaque barre représente une valeur moyenne, calculée sur au moins 7 portées, \pm son écart type. Les valeurs sont comparées à l'aide du test $t$ de Student Fischer ; pour les valeurs statistiquement différentes, les niveaux de signification sont les suivants : ${ }^{*} P<0,05 ;{ }^{* *} P<0,001$.

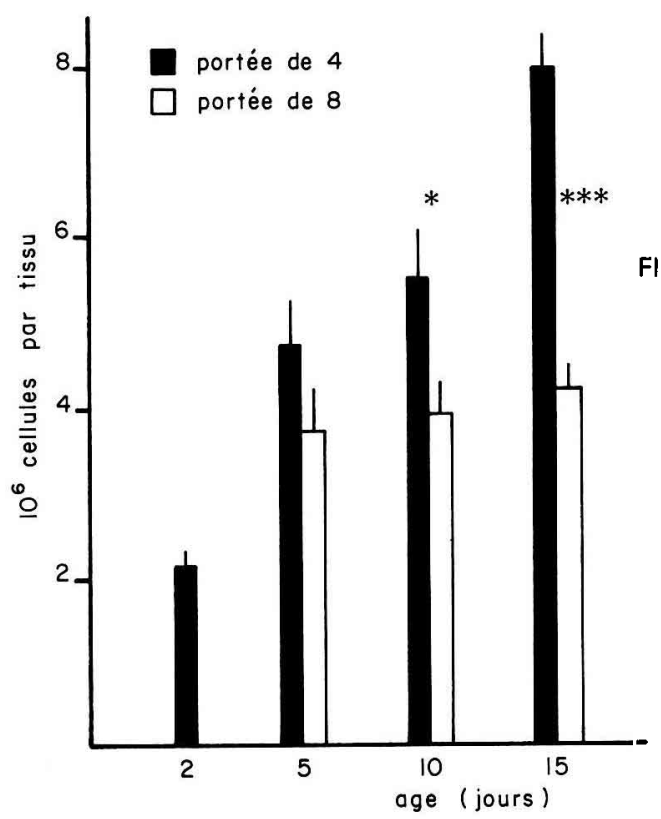

FIG. 3. - Effet de la suralimentation précoce sur le nombre de cellules dans la fraction stromavasculaire du tissu inguinal. Chaque barre figure une valeur moyenne calculée sur au moins 7 portées, assortie de son écart type. Les valeurs obtenues pour les animaux élevés par portées de 4 sont comparées à celles obtenues pour les animaux élevés par portées de 8 par le test $t$ de Student Fischer. Pour les valeurs statistiquement différentes, les niveaux de signification sont les suivants : ${ }^{*} P<0,05$; $* * * P<0,001$. 
d'âge, et il n'y a pas de différence de ce nombre entre les rats élevés par portées de 4 et ceux élevés par portées de 8 ; à 15 jours d'âge, par contre, les rats suralimentés présentent une hyperplasie adipocytaire. Chez le témoin le nombre de cellules dans la fraction stroma-vasculaire du tissu inguinal (fig. 3) est doublé entre 2 et 5 jours, et reste stable par la suite. Par contre, chez les animaux suralimentés, ce nombre augmente régulièrement au cours du développement, de telle sorte que, à partir de l'âge de 10 jours, il y a significativement plus de cellules dans le compartiment stroma-vasculaire des rats suralimentés comparés aux témoins.
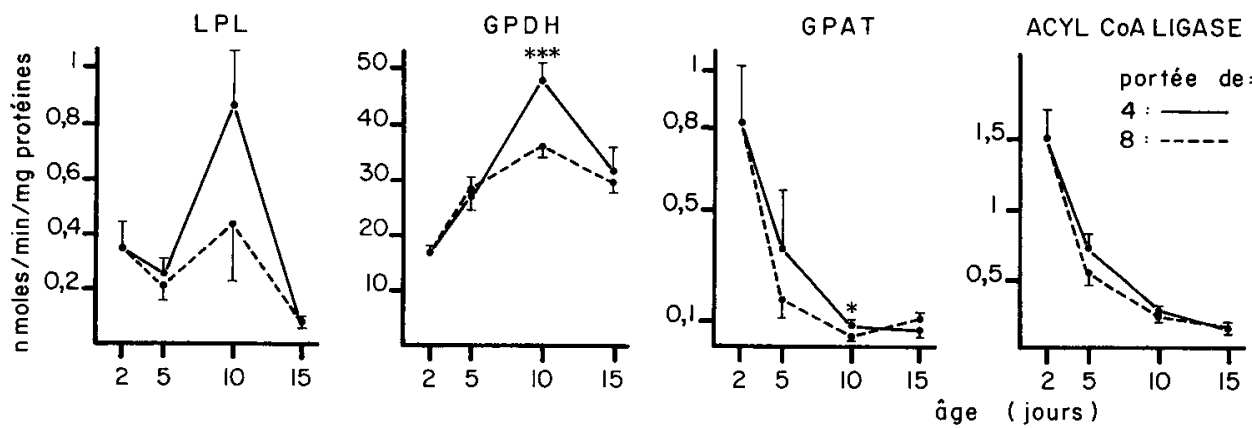

FIG. 4. - Effet de la suralimentation précoce sur les activités enzymatiques des cellules de la fraction stroma-vasculaire du tissu inguinal.

Partie supérieure: activité de la lipoprotéine lipase (à gauche), et de la glycérol-3-Phdéshydrogénase (à droite);

Partie inférieure: activité de la glycéro-Ph-acyl-transférase (à gauche) et de la acyl-CoA-ligase (à droite).

Chaque point représente une valeur moyenne, calculée sur au moins 4 portées, assortie de son écart type. Les valeurs sont comparées à l'aide du test $t$ de Student Fischer. Les niveaux de signification sont les suivants : ${ }^{*} P<0,05 ; * * * P<0,001$.

L'évolution des activités de la LPL, GPDH, GPAT, et acyl CoA ligase dans les cellules de la fraction stroma-vasculaire est représentée sur la figure 4 . Chez les témoins, les activités de la LPL et de la GPDH sont faibles dans les premiers jours de la vie, augmentent ensuite pour atteindre un pic d'activité à l'âge de 10 jours ; les activités de la GPAT, et de l'acyl CoA ligase sont maximales 2 jours après la naissance et décroissent ensuite régulièrement pendant la période d'expérimentation.

Chez les animaux suralimentés, le profil d'évolution au cours du développement des différentes activités enzymatiques n'est pas modifié. Toutefois, à l'âge de 10 jours, on note une augmentation significative des activités GPDH et GPAT chez les animaux surnourris. A cet âge, l'activité de la LPL des rats suralimentés est doublée par rapport aux témoins mais les valeurs ne sont pas statistiquement différentes. A l'âge de 15 jours, il n'y a plus de différence entre les 2 groupes d'animaux quelle que soit l'enzyme considérée. 


\section{Discussion.}

En accord avec tous les résultats précédents, notre expérience montre un effet rapide de la suralimentation précoce, à la fois sur la vitesse de croissance et sur le développement du tissu adipeux (Kennedy, 1957 ; Widdowson et Mac Cance, 1960 ; Cryer et Jones, 1979 ; Wurtman et Miller, 1976 ; Duff et Snell, 1982). En effet, que la suralimentation dès les premiers jours de la vie soit induite par réduction de la taille des portées, ou par infusion intragastrique continue d'une solution proche du lait maternel (West et al., 1982), le poids des animaux suralimentés au moment du sevrage est toujours supérieur à celui des témoins. Au cours du développement du tissu adipeux inguinal, la comparaison des animaux élevés par portée de 4 et de leurs témoins élevés par portée de 8 montre que la suralimentation provoque à court terme une hypertrophie des adipocytes et à " long terme » une hyperplasie adipocytaire.

Ces résultats nous montrent que les 2 composantes du surdéveloppement tissulaire, caractéristiques de cette obésité à l'âge adulte (Knittle et Hirsh, 1968 ; Cryer et Jones, 1980 ; Faust et al., 1980), sont déjà présentes avant le sevrage. Nous voyons donc que avant toute manifestation d'obésité, le surdéveloppement précoce du tissu adipeux dans ce modèle est principalement lié à une hypertrophie adipocytaire qui serait ainsi une des premières manifestations de cette obésité comme dans le cas de l'obésité génétique du rat Zucker (Boulangé et al., 1979). Au niveau du compartiment stroma-vasculaire, la suralimentation provoque des modifications dans la prolifération cellulaire et la capacité de différenciation des cellules stromales. Nos résultats montrent que chez les animaux suralimentés il existe une prolifération plus active que chez les témoins, aboutissant à terme à une augmentation du nombre des cellules dans le compartiment adipocytaire. Ces résultats sont à rapprocher de ceux obtenus par Gaben-Cogneville et al. (1981), qui ont établi que le niveau des ingesta pendant les premiers jours de la vie pouvait réguler la multiplication cellulaire et la vitesse de différenciation dans le tissu inguinal. Dans cette expérience en effet, ces auteurs en suivant l'incorporation de thymidine tritiée dans le DNA, ont montré que la sous-alimentation précoce diminue à la fois la vitesse de replication des cellules et leur capacité de différenciation. Après 10 jours de déprivation, ces altérations deviennent irréversibles. Ces résultats et les nôtres soulignent l'importance, pour le développement du tissu adipeux d'une période dite " critique » vers l'âge de 10 jours.

Aucune donnée n'a encore établi, à notre connaissance, le profil d'évolution dans les cellules stromales des activités enzymatiques caractéristiques de la fonction adipocytaire au cours du développement. Nos résultats indiquant, dans ces cellules, un pic d'activité de la LPL à l'âge de 10 jours, sont à rapprocher de ceux obtenus par Cryer et Jones (1979) dans le tissu entier. D'une façon générale, la suralimentation provoque une stimulation des activités enzymatiques caractéristiques de la fonction adipocytaire dans les cellules stromales, sans jamais altérer le profil d'évolution de ces activités au cours du développement. Ceci suggère que le processus ontogénique de développement du tissu n'est pas modifié, mais simplement amplifié par la suralimentation. II est vraisemblable que l'insuline soit en par- 
tie responsable de cette amplification des activités. En effet Cryer et Jones ont montré que pendant la période de suralimentation les animaux élevés dans des portées de petites tailles ont une insulinémie supérieure à celle des témoins. II apparaît donc, à l'âge de 10 jours, une surcapacité de différenciation d'autant plus importante qu'il y a à cet âge davantage de cellules stromales. Néanmoins, la fraction stroma-vasculaire du tissu adipeux est hétérogène, non seulement du point de vue des types cellulaires mais aussi du point de vue du degré d'avancement des cellules souches dans la voie de différenciation. II est donc difficile de dire si les tissus des animaux suralimentés contiennent davantage de cellules ayant entamé le processus de conversion adipocytaire ou bien si ces cellules sont plus avancées dans la voie de la différenciation.

\section{Conclusion.}

La suralimentation précoce, en provoquant à la fois une prolifération cellulaire et une augmentation du potentiel de différenciation dans les cellules de la fraction stroma-vasculaire, induit une réelle surcapacité de stockage du tissu adipeux. Chez les animaux suralimentés, cette surcapacité de stockage se traduit, outre l'hypertrophie par une hyperplasie adipocytaire qui persiste à l'âge adulte.

Cette étude ne permet pas de dire quels sont les facteurs agissant au niveau du compartiment stromal et impliqués dans l'accélération du processus de différenciation chez les jeunes rats suralimentés. II est toutefois vraisemblable que ces facteurs doivent être reliés, directement ou non, à la présence d'une hyperphagie.

10e Réunion du groupe Développement I.N.R.A., Rennes, 9-10 mai 1984.

Remerciements. - Ce travail a été subventionné par l'Institut National de la Santé et de la Recherche Médicale (CRL $n^{\circ}$ 82.70.22). - Les auteurs remercient Melle V. Resve qui a assuré la dactylographie de cet article.

\section{Références}

BOULANGÉ A., PLANCHE E., de GASQUET P., 1979. Onset of genetic obesity in the absence of hyperphagia during the 1st week of life in Zucker rat (fa/fa). J. Lipid Res., 20, 857-864.

CRYER A., JONES H. M., 1979. The early development of white adipose tissue. Biochem. J., 178, 711-724.

CRYER A., JONES H. M., 1980. The development of white adipose tissue. Biochem. J., 86, 805815.

DJIAN P., RONCARI D. A. K., HOLLENBERG C. H., 1983. Influence of anatomic site and age on the replication and differentiation of rat adipocyte precursor in culture. J. clin. Invest,, 72 , 1200-1208.

DUFF D. A., SNELL K., 1982. Effect of altered neonatal nutrition on the development of enzymes of lipid carbohydrate metabolism in the rat. J. Nutr., 112, 1057-1066. 
FAUST I. M., JOHNSON P. R., HIRSCH J., 1980. Long term effects of early nutritional experience on the development of obesity in the rat. J. Nutr., 110, 2017-2034.

FOLCH J., LEES M., SLOANE-STANLEY G. H., 1957. A simple method for isolation and purification of total lipids from animals tissue. J. biol. Chem., 226, 497-509.

GABEN-COGNEVILLE A. M., JAHCHAN T., SWIERCZEWSKI E., 1981. Alteration by early underfeeding of cellular multiplication and differentiation in the inguinal fat pads of rats. J. Nutr., 111, 2098-2105.

HAUSMAN G. J., CAMPION D. R., MARTIN R. J., 1980. Search for the adipocyte precursor cell and factors promote its differentiation. J. Lipid Res., 21, 657-670.

JAMDAR S. C., FALLON H. J., 1973. Glycerolipid synthesis in rat adipose tissue. J. Lipid Res., 14, 509-510.

KENNEDY G. C., 1957. The effect of age on the somatic and visceral response to overnutrition in the rat. J. Endocrinol., 15, 19-24.

KNITTLE J. L., HIRSCH J., 1968. Effect of early nutrition on the development of rat epididymal fat pads : cellularity and metabolism. J. clin. Invest., 47, 2091-2098.

KOZAK L. P., JENSEN J. T., 1974. Genetic and developmental control of multiple forms of L glycerol 3-phosphate deshydrogenase. J. biol. Chem., 249, 7775-7781.

LOWRY O. H., ROSEBROUGH N. J., FARR A. L., RANDALL R. J., 1951. Protein measurement with the folin phenol reagent. $J$. biol. Chem., 193, 265-271.

NILSSON-EHLE P., SCHOTZ M. C., 1976. A stable radioactive substrate emulsion for assay of lipoprotein lipase. J. Lipid Res., 17, 536-541.

OSCAI L. B., Mc GARR J. A., 1978 . Evidence that amount of food consumed in early life fixes appetite in the rat. Am. J. Physiol., 235 (3), R141-R144.

ROTHBLATT G. H., de MARTINIS F. D., 1977. Release of LPL from rat adipose tissue cells grown in culture. Biochem. biophys. Res. Commun., 78, 45-50.

TANAKA T., HOSAKA K., NUMA S., 1979. Long chain acyl-CoA synthetase from rat liver. In LOWENSTEIN J. M. Methods of Enzymology, vol. 71. 334, Acad. Press.

WEST D. B., DIAZ J., WOOD S. C., 1982. Infant gastronomy and chronic formula infusion as a technique to overfeed and accelerate weight gain in neonatal rats. J. Nutr., 112, 1339-1343.

WIDDOWSON E. M., Mac CANCE R. A., 1960. Some effects of accelerating growth. I General somatic development. Proc. roy Soc. London, B., 152, 188-206.

WISE L. S., GREEN H., 1979. Participation of one isoenzyme of cytosolic glycerophosphate deshydrogenase in the adipose conversion of 3T3 cells. J. biol. Chem., 254, 373-375.

WURTMAN J. J., MILLER S. A., 1976. Effect of litter size on weight gain in rats. J. Nutr., 103, 738-743. 